\title{
From cutting-edge pointwise cross-section to groupwise reaction rate: A primer
}

\author{
Jean-Christophe Sublet ${ }^{\mathrm{a}}$, Michael Fleming, and Mark R. Gilbert \\ United Kingdom Atomic Energy Authority, Culham Science Centre, Abingdon, Oxfordshire OX14 4BD, UK
}

\begin{abstract}
The nuclear research and development community has a history of using both integral and differential experiments to support accurate lattice-reactor, nuclear reactor criticality and shielding simulations, as well as verification and validation efforts of cross sections and emitted particle spectra. An important aspect to this type of analysis is the proper consideration of the contribution of the neutron spectrum in its entirety, with correct propagation of uncertainties and standard deviations derived from Monte Carlo simulations, to the local and total uncertainty in the simulated reactions rates (RRs), which usually only apply to one application at a time. This paper identifies deficiencies in the traditional treatment, and discusses correct handling of the RR uncertainty quantification and propagation, including details of the cross section components in the RR uncertainty estimates, which are verified for relevant applications. The methodology that rigorously captures the spectral shift and cross section contributions to the uncertainty in the RR are discussed with quantified examples that demonstrate the importance of the proper treatment of the spectrum profile and cross section contributions to the uncertainty in the RR and subsequent response functions.

The recently developed inventory code FISPACT-II, when connected to the processed nuclear data libraries TENDL-2015, ENDF/B-VII.1, JENDL-4.0u or JEFF-3.2, forms an enhanced multi-physics platform providing a wide variety of advanced simulation methods for modelling activation, transmutation, burnup protocols and simulating radiation damage sources terms. The system has extended cutting-edge nuclear data forms, uncertainty quantification and propagation methods, which have been the subject of recent integral and differential, fission, fusion and accelerators validation efforts. The simulation system is used to accurately and predictively probe, understand and underpin a modern and sustainable understanding of the nuclear physics that is so important for many areas of science and technology; advanced fission and fuel systems, magnetic and inertial confinement fusion, high energy, accelerator physics, medical application, isotope production, earth exploration, astrophysics and homeland security.
\end{abstract}

\section{Introduction}

In lattice-reactor simulation the aim is to accurately model the neutron flux map, follows the inventory of the major fissile isotopes and a few fission products to account for $K_{\text {eff }}$ variation in fuel cycle. An important aspect of the fission reactor specificity is that it invariably has a lattice geometry, with regularly distributed source terms, but in the reactor edges. Radiation shielding also need to simulate well the neutron maps, but then across regions of diverse impact, where it decrease and it energy distribution shift significantly. The emphasis to model neutron slowing down in matter is then driven by the specific engineering needs and will have an impact on the simulation method chosen. This often allows modelling short-cut, simplification, optimisation and targeting to be made to benefit the responses needed for particular application. It is not uncommon to subsequently not been able to derived further responses with the same accuracy as were the first set. To properly capture a reaction rate a delicate balance need to be found that encompasses two correlated quantities: reaction cross section and particle or neutron flux. Both quantities are energy dependent with peak and trough usually of inverted sense in identical

a e-mail: jean-christophe.sublet@ukaea.uk energy ranges. A representative neutron flux profile can vary rapidly within a cell and abruptly at material regions boundaries, such as coolant channel, while different reaction channels of the same isotope of a given cell or region been emphasised or belittle by different energy regions of the neutron spectra. It is also fair to say that not only the energy and spatial dimensions are of some importance but also the temporal one.

\section{Particle spectra}

The simulation of neutron spectra at any spatial position is carried out by the transport code. Modellers have a choice between using a deterministic or Monte Carlo solver, however this choice is not without impact on the "accuracy" of the solution. This is mainly due to the fact that both methods have to rely on processed, through different methods, basis nuclear data forms. The Monte Carlo method been the most physical, if and only if all convergences criteria have been achieved, this is rarely the case. Deterministic method are groupwise from the start but the energy bins of the group structure they rely upon to simulate the neutron map has been carefully selected with regard to operational criteria that are not general purpose. At the dawn of the fission industry 


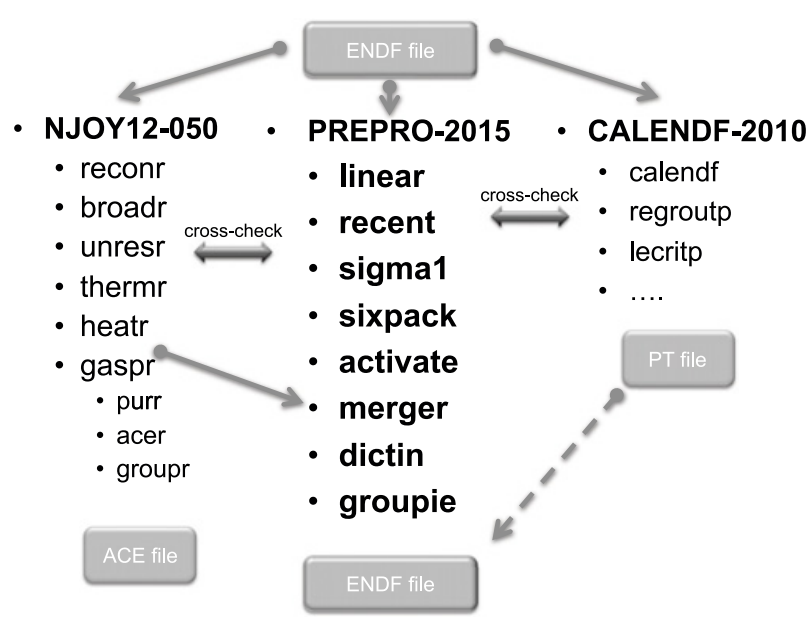

Figure 1. Schematic of the processing sequence using NJOY, PREPRO and CALENDF.

reactors simulation were performed on a coarse energy grid: typically 69 groups for the lattice code WIMS and then up to only $10 \mathrm{MeV}$. However, nowadays technologies allows the simulation to seamlessly climb to finer grid of a few hundreds, one thousand group allowing the capture of many more fine structures.

It is important to keep in mind that it is in fine, the neutron flux profile detail in the most important energy region of importance for a given reaction rates that will set the floor of the accuracy expected on the reaction rate. So the finer the structure the better, particularly in the resonance regions.

Typical neutron flux profile for four reactor type are shown in Fig. 4. One can see that there are more above $14 \mathrm{MeV}$ neutron in fission types reactors than in the first wall of a conceptual fusion demonstration reactor. The legacy PWR spectra (EDF Paluel) also show an earlier specificity: the simulation had a cut-off at $10 \mathrm{MeV}$. This has no influence on the reactor operations, but could be detrimental to any subsequent fuel inventory simulation for wet or dry storage.

\section{Multigroup constants}

The preparation of nuclear data from diverse sources for use in FISPACT-II [1] was streamlined and simplified by following as closely as possible the forms of data format described in the ENDF-6 format manual [2].

Three processing codes are used in sequence and in parallel to produce, process, check, and compare the various original nuclear data forms: NJOY12-050 [3], PREPRO-2015 [4] and CALENDF-2010 [5]. No one, or even two, of these processing codes are sufficient to manage all the required steps. In general, a combination of the three is needed to extract the multi-physics data forms that are used to create the most versatile library files for FISPACT-II. A schematic of the processing sequence is shown in Fig. 1.

Further details of the data assimilation processes and their history can be found in $[6,7]$. TENDL processed data forms differ in some respects [8,9]. This is due to often subtle enhancements made in the original but completely ENDF-6 compliant TENDL data format and the way the files themselves are processed. This is particularly

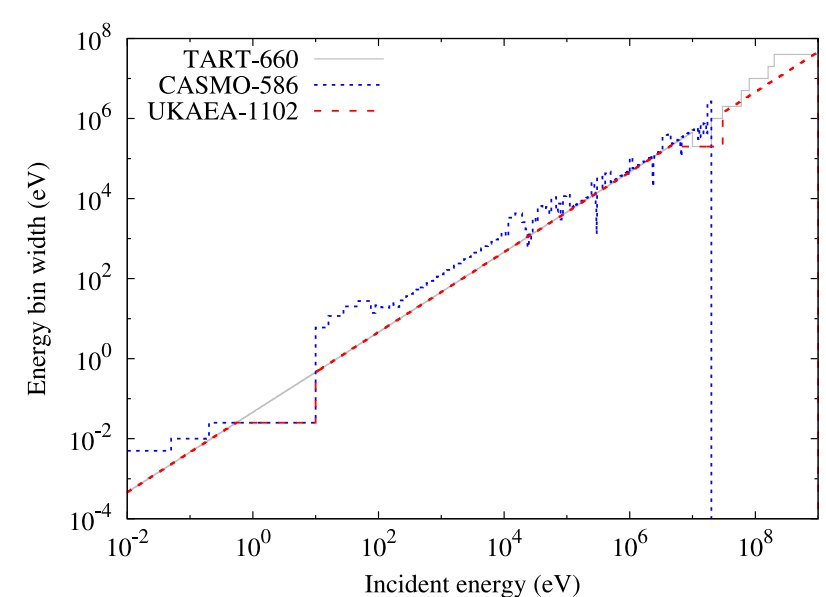

Figure 2. Energy bin widths for the TART-660, CASMO-586 and UKAEA-1102 group structures.

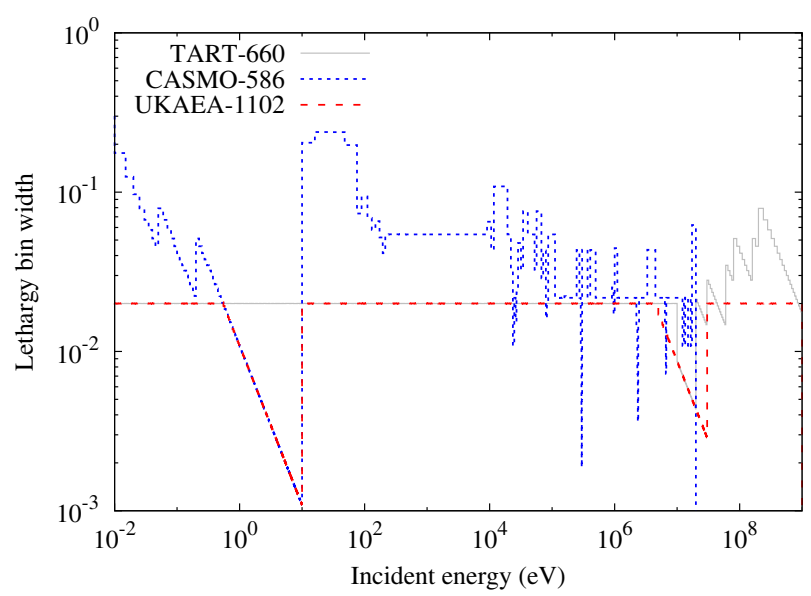

Figure 3. Lethargy bin widths for the TART-660, CASMO-586 and UKAEA-1102 group structures.

noticeable in the partials for kerma, dpa and PKA generated by the latest TALYS and the more complete use made of the variance and covariance information contained in this truly general purpose library.

\subsection{Cross section group structure}

There are three standard group structures in the ENDF-6 format used for the TENDL, ENDF/B.VII.1, JENDL-4.0, CENDL-3.1 and JEFF-3.2 nuclear libraries; data in these structures can be read automatically into FISPACT-II. They have a fine energy grid, an increased upper energy bound of $1 \mathrm{GeV}$, and allow the addition of $\alpha$ and $\gamma$-induced reactions while permitting more precise modelling of reaction thresholds and the resolved and unresolved resonance ranges. These groups are:

\begin{tabular}{lc}
\hline \hline Name & Number of groups \\
\hline CCFE & 162 \\
CCFE & 709 \\
UKAEA & 1102 \\
\hline
\end{tabular}

The method of presentation in Fig. 2 (energy bin width) and Fig. 3 (lethargy bin width), is designed to make clear in which energy ranges particular structures have most groups and will therefore give a good, accurate representation of the reaction rates. 


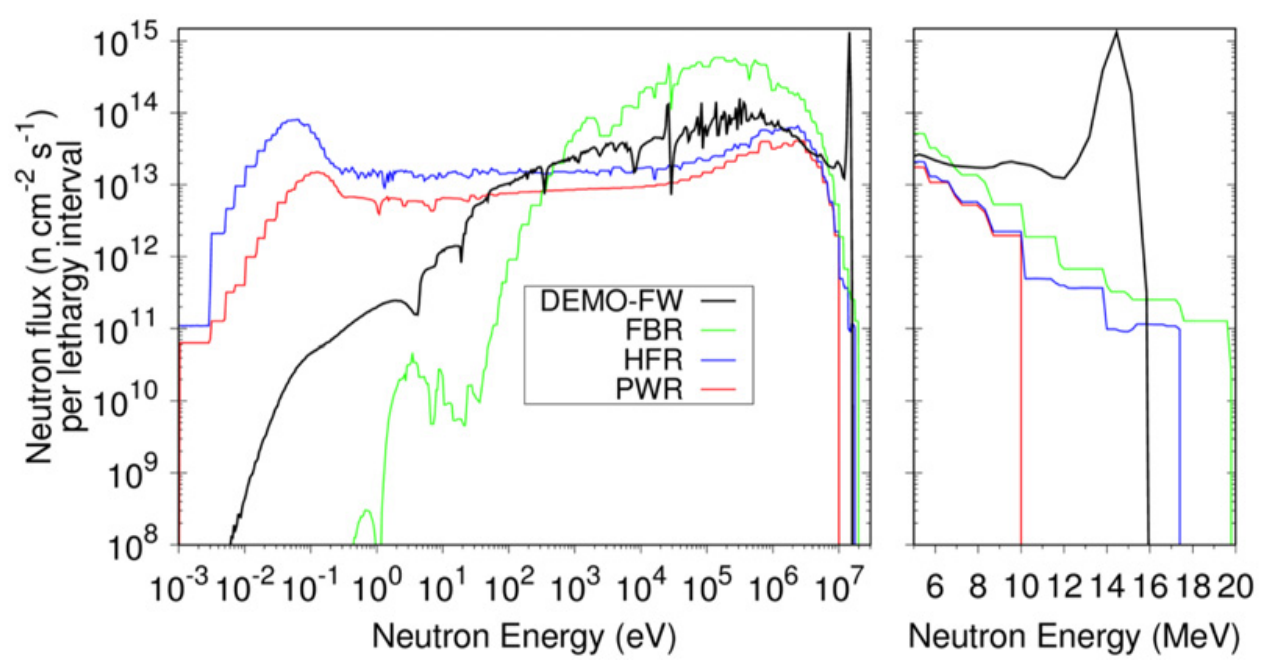

Figure 4. Typical neutron flux spectra for different reactors types: Fusion, Fast breeder, High flux and Light water.

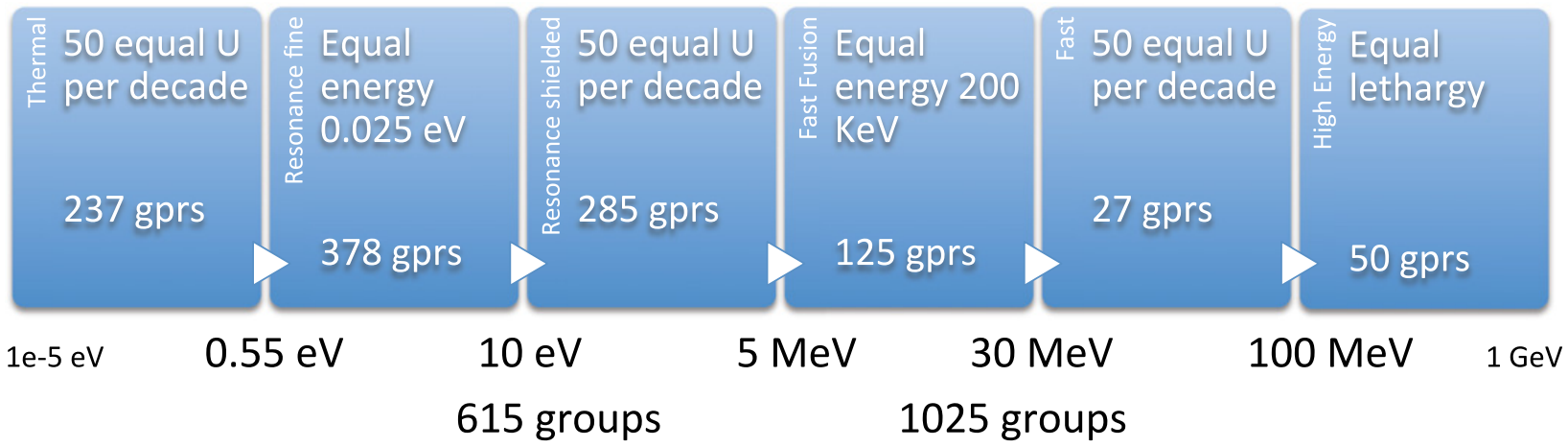

Figure 5. UKAEA-1102 group structures description.

The CCFE-162 (up to $200 \mathrm{MeV}$ ) structure was introduced for studies of charged-particle projectiles and $\gamma$-induced activation and transmutation. This structure includes all legacy ones known to the author, because past limitations of computing resources are no longer a consideration justifying simpler energy structures. The CCFE (709 up to $200 \mathrm{MeV}$ and 660 up to $30 \mathrm{MeV}$ ) group structures are extensions of the LLNL (616 up to $20 \mathrm{MeV}$ ) structure. They have 50 tally bins per energy decade, equally spaced in the logarithm of the energy between $10^{-5} \mathrm{eV}$ and $10 \mathrm{MeV}, 200 \mathrm{keV}$ steps to $30 \mathrm{MeV}$ and thereafter bins with appropriately chosen equallyspaced boundaries in energy up to $1 \mathrm{GeV}$. The UKAEA 1102-group structure is the culmination of extensions of the state of the art thermal fission CASMO (586), the fast LLNL (616) and the fast CCFE (709) structures pushed to $1 \mathrm{GeV}$. Each specific feature of those three grids have been combined into one single structure: the UKAEA 1102group structure depicted in Fig. 5.

The generation of reaction rates with multi-group convolution of binned fluxes with cross-sections has always been the subject of intense research in order to satisfy the specific requirements of one application at a time. Recent studies $[10,11]$ have demonstrated that the fine CCFE-709 and a fortiori the UKAEA-1102 group structure have been optimised to comprehensively cover all applications.

\subsection{Reaction rates}

A sample of Tungsten was irradiated at the high flux reactor HFR in Petten under the exercise EXTREMAT-II in 2008-1008 for 8 cycles and at two different positions. The sample was positined next to another experiment with very strong thermal neutron absorption properties. This occasioned the $\mathrm{W}$ sample to be exposed to a lower than normal (for HFR) fraction of thermal neutrons. The spectral shifts occasioned are depicted in Fig. 8 were the local $\mathrm{C} 3$ and $\mathrm{C} 7$ position thermal maxwellian can be seen to be less prominent.

The simulated HFR neutron flux profiles are represented in the fine 709 structure and this allows the collapsed reaction rates to properly account for the spectral shifts that would otherwise not have been considered for important or been noticed.

It is noticeable that in the case of the $186 \mathrm{~W}$ capture reactions almost $50 \%$ of the RR comes from neutron of energy below $10 \mathrm{eV}$, while for the $184 \mathrm{~W}$ capture this "threshold" is below 100, or $200 \mathrm{eV}$ dependant on the position and or HFR original neutron spectra.

This too often forgotten aspect, that the reaction rates is a collapse of the cross section and the flux, that sometimes emphasis strongly the $1 / \mathrm{E}$, or giant resonance region may lead to significant overestimation of the reaction rates when and if

- the neutron flux grid is too coarse;

- the simulation do not account of the local heterogeneity;

- the giant resonance region is not properly represented.

It should also be clear that the same impediment (grid mismatch) would have the same deleterious effects in the 


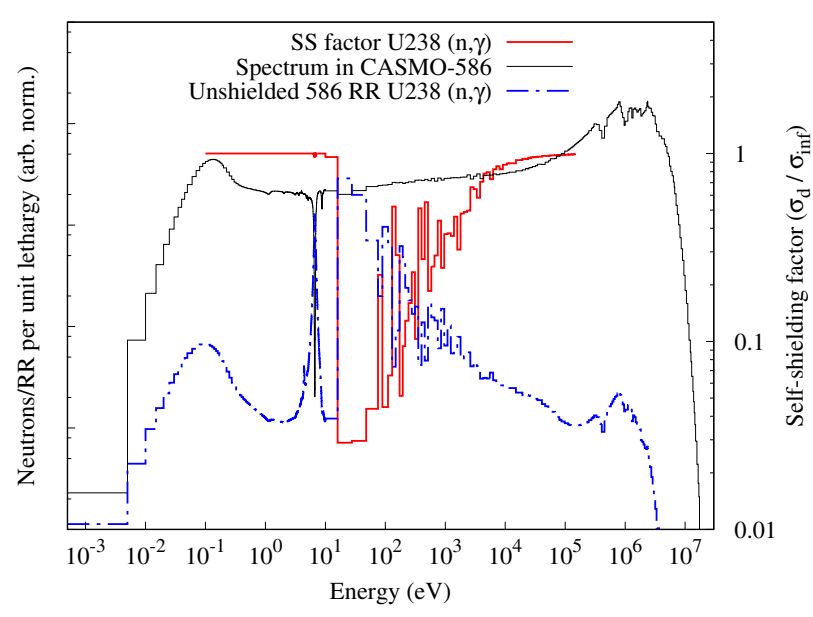

Figure 6. Neutron spectrum, the unshielded 238-U cross sections and the energy-dependent probability table self-shielding factors.

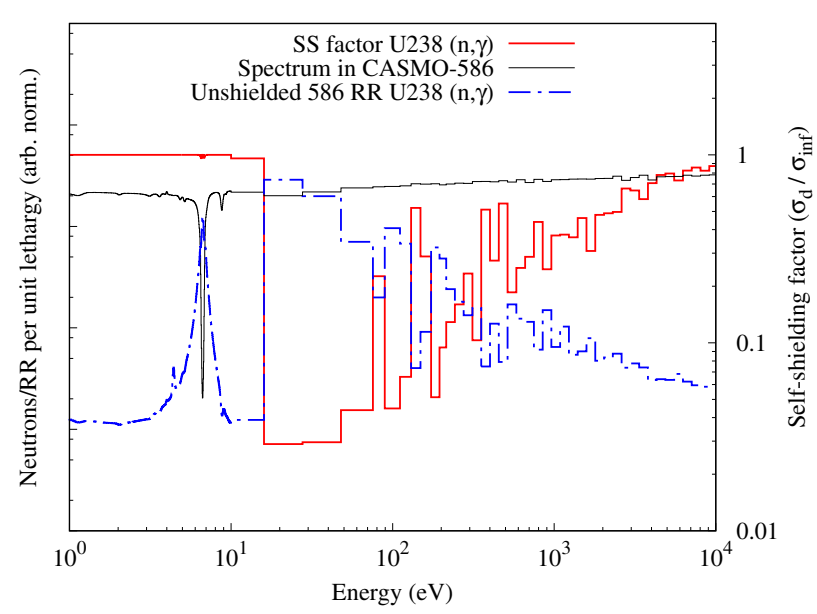

Figure 7. Figure 5 zoomed.

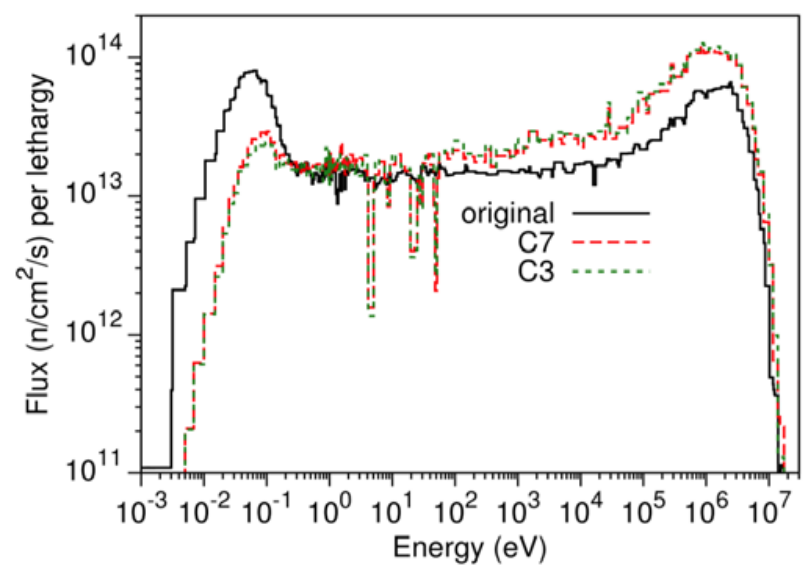

Figure 8. HFR Petten spectra.

energy region of the threshold of negative $\mathrm{Q}$ reactions channels.

\section{Summary}

The FISPACT-II system brings many new capabilities to the long established family of inventory codes. At the core of the main code is a modern rate equation solver that exploits the most advanced physics provided in modern

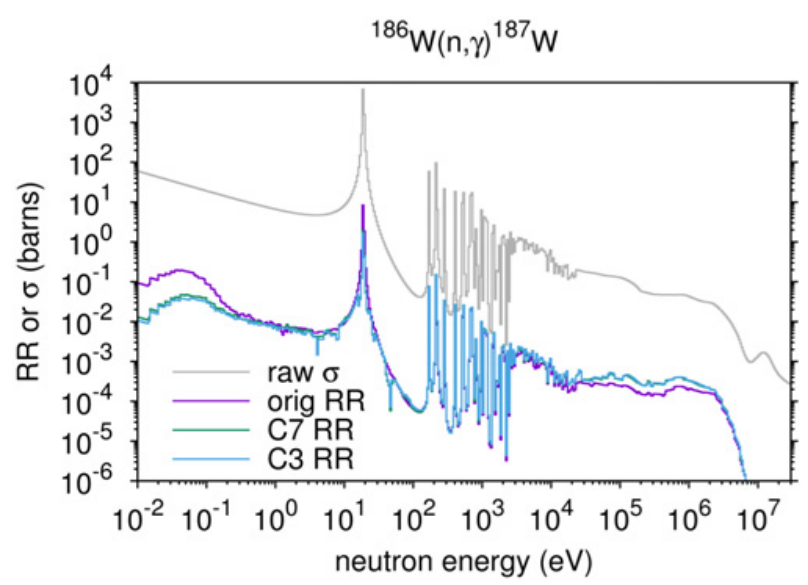

Figure 9. HFR Petten 186-W cross sections and reactions rates.

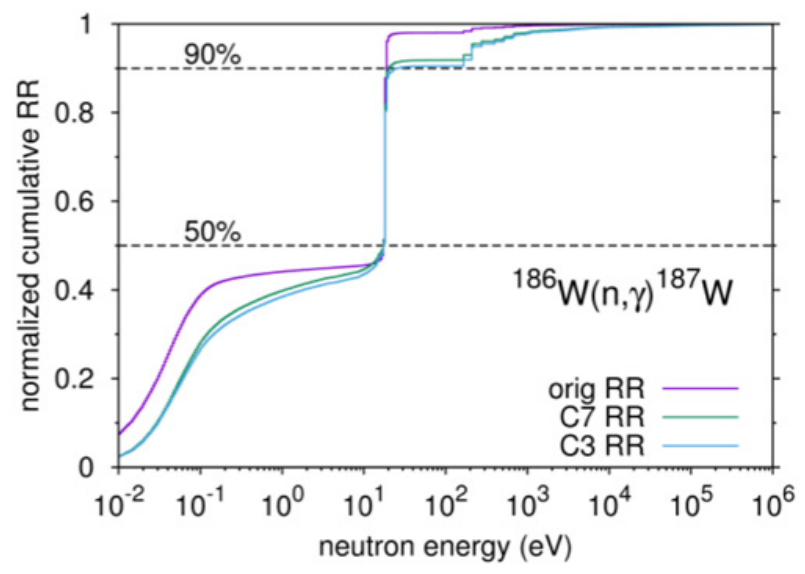

Figure 10. HFR Petten 186-W normalised reactions rates.

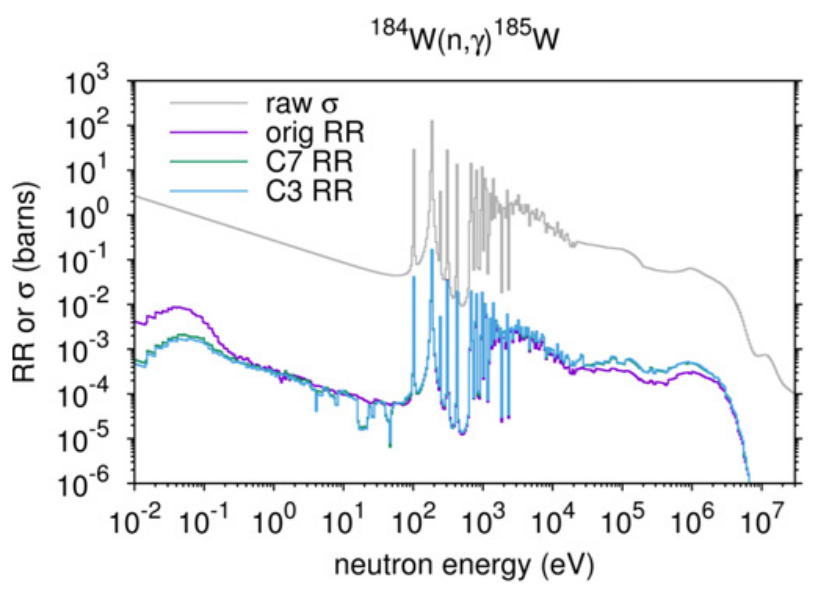

Figure 11. HFR Petten 184-W cross sections and reactions rates.

nuclear data forms. This has primarily been driven by the development of the technological TENDL nuclear data libraries that, when coupled with FISPACT-II, allow truly general-purpose simulations for neutron-induced inventory calculations, as well as a charged-particle simulations for proton, deuteron, alpha and gamma-ray irradiation.

The use of modern nuclear data forms opens up many novel features. New data forms for probability tables offer adaptable self-shielding calculations for any isotopic composition of materials and any irradiation scenarios, as well as geometry-specific self-shielding for several configurations. The inclusion of all (partial 


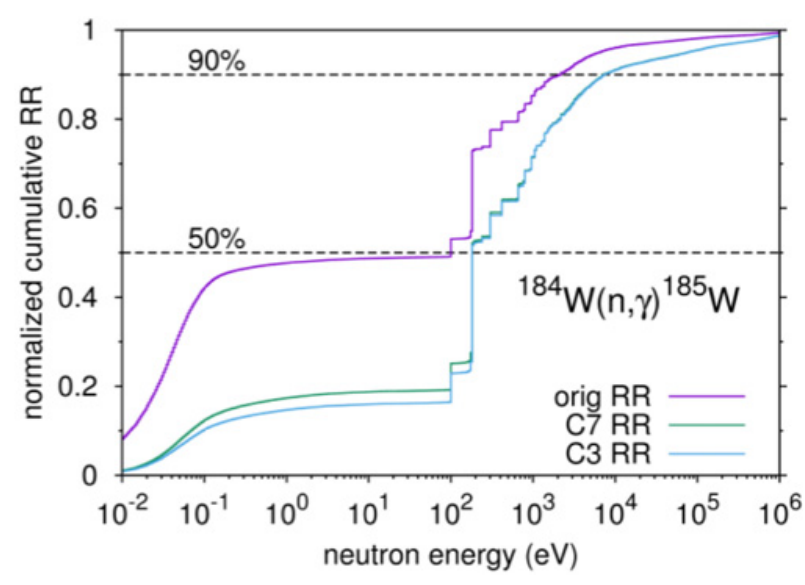

Figure 12. HFR Petten 184-W normalised reactions rates.

and total) reaction channels offers robust simulation of all inventory response functions, including source terms, gamma-dose, decay heat, activation-transmutation, depletion, gas production and dpa. The availability of complete covariance data for all reaction channels, at least in TENDL, combined with the sophisticated pathwaysbased and Monte-Carlo sensitivity methods of FISPACT-II offers novel uncertainty quantification and propagation methods for all physical systems. The high-energy residual nuclide production data of TENDL, as read by FISPACT-II, allows fully tabulated nuclear data to be used in simulations of incident particles up to several hundred $\mathrm{MeV}$.

Accessing all ENDF-6 nuclear data files, including both the well-known international libraries and the modern, technological files such as TENDL and GEFY has allowed FISPACT-II to be used to perform rigorous testing of the data against experimental values. These have verified the code capabilities, validated simulation results and offerer a new method for probing the nuclear landscapes out of the regions where experimental data exists.
This work was funded by the RCUK Energy Programme [grant number EP/I501045]. To obtain further information on the data and models underlying this paper please contact PublicationsManager@ccfe.ac.uk

\section{References}

[1] J.C. Sublet, J. Eastwood, J. Morgan, M. Fleming, M. Gilbert, Tech. Rep. UKAEA-R(11)11 Issue 7, UKAEA (2015), http://www.ccfe.ac.uk/fispact.aspx

[2] M. Herman, A. Trkov, eds., ENDF-6 Formats Manual, Data Formats and Procedures for the Evaluated Nuclear Data File ENDF/B-VI and ENDF/B-VII, Vol. BNL-90365-2009 Rev. 2 (Brookhaven National Laboratory, 2011)

[3] R.E. MacFarlane, D.W. Muir, R.M. Boicourt, A.C. Kahler, The NJOY Nuclear Data Processing System LA-UR-12-27079 (Version 2012-032), http: //t2 . lanl.gov/nis/publications/NJOY2012.pdf

[4] D. Cullen, Tech. Rep. IAEA-NDS-39 (Rev. 16), IAEA (2015), http://www-nds.iaea.org/ ndspub/endf/prepro/

[5] J.C. Sublet, P. Ribon, M. Coste-Delclaux, Tech. Rep. CEA-R-6277, ISSN 0429-3460, CEA (2011)

[6] J.C. Sublet, A. Koning, R.A. Forrest, J. Kopecky, Tech. Rep. JEFFDOC-982, NT DER/SPRC/LEPH 03/220, OECD, CEA (2003)

[7] J.C. Sublet, A. Koning, D. Rochman, Tech. Rep. CCFE-R(11)16, CCFE (2012)

[8] A. Koning, D. Rochman, Nuclear Data Sheets 113, 2841 (2012)

[9] D. Rochman, A.J. Koning, J. Kopecky, J.-Ch. Sublet, P. Ribon, M. Moxon, Annals of Nuclear Energy 51, 60 (2013)

[10] A. Hodgson, R. Grimes, M. Rushton, O. Masden, Nuclear Science and Engineering 181, 1 (2015), http://dx.doi.org/10.13182/NSE14-156

[11] M. Fleming, L. Morgan, E. Shwageraus, Nuclear Science and Engineering 183, 179 (2016), http://dx.doi.org/10.13182/NSE15-55 